\title{
Less is more: a project to reduce the number of PIMs (potentially inappropriate medications) on an elderly care ward
}

\author{
Tin Htun Aung, Adèle Judith Beck, Thomas Siese, Richard Berrisford \\ Derriford Hospital, Plymouth hospitals NHS trust, UK
}

\begin{abstract}
Potentially inappropriate prescribing in healthcare of the elderly (HCE) is associated with avoidable adverse drug events (ADEs).[1,2] A recent set of prescribing criteria has been designed and validated, called "Screening Tool of Older Persons' Prescriptions" (STOPP), to rationalise prescribing in hospitalised patients on HCE wards.[1,3] The aim of this quality improvement project was to identify how many potentially inappropriate medications (PIMs) were prescribed on these wards, and remove them. This was executed by implementing a ward round checklist, which incorporated STOPP criteria, for the twice weekly, consultant led ward rounds.
\end{abstract}

This quality improvement project was carried out over four months. In a pilot study, we identified eight inappropriate medical prescriptions among 148 medications (5.4\%) prescribed on one ward. After applying a checklist for a structured ward round, we reviewed the medications prescribed on that ward, and found 10 PIMs out of 192 (5.2\%). Utilising the increasingly recognised "plan, do, study, act" (PDSA) cycle,[4] we implemented departmental teaching and meetings with other members of the multidisciplinary team, which raised awareness of PIMs among junior doctors, as well as involving our pharmacists in drug chart screening. During this process we continued with a further six cycles on a biweekly basis, and saw a gradual decrease in PIM to $1.5 \%$.

In conclusion, a structured ward round, facilitated by a checklist that included review of drug charts based on STOPP criteria, demonstrated a considerable reduction of PIMs. It would be interesting to apply this quality improvement project to non-HCE wards, including general surgical wards or adult psychiatry wards, as a means of not only reducing the effects of ADEs, but also the expenditure associated with unnecessary drug prescriptions, and the costs associated with additional care arising from associated ADEs.

\section{Problem}

Elderly patients are particularly vulnerable to complications arising from adverse drug events (ADEs) and polypharmacy, due to their higher burden of multiple comorbidities,[2] declining health and social welfare, and deteriorating cognitive function.[5] While some medications are justified through reducing the risks of harms in terms of mortality and morbidity, others arguably pose more harm than good. It is often easier as a clinician to commence a patient on a new drug for several valid reasons at a specific moment, than reviewing and stopping medication further down the line. As junior doctors on HCE rotations at Derriford Hospital, Plymouth, UK, clerking in elderly patients on the medical assessment unit, as well as reviewing such patients on our wards, we encountered numerous occasions where patients were on multiple medications, with potential harms posed by drug interactions and side effects.

The risk/benefit ratio is dynamic in a temporal sense, but also in relation to the health problems which affect the patient. Proactive review of elderly patients' medications is therefore justified. Hospitalisation presents an important opportunity to review medications from both short and long term perspectives. Does the patient still benefit from the prescribed medication? Or are they suffering from subclinical or hitherto undiagnosed side effects? A large number of inappropriate medications have been highlighted in this area, and while they may lead to significant iatrogenic effects, they are far too often discovered on drug prescriptions of elderly people with no appropriate justification. This has led to development of STOPP criteria to improve prescribing in the elderly, and reduce the harms associated with ADEs and polypharmacy.

\section{Background}

Potentially inappropriate medications (PIMs) prescribed in HCE are shown to be associated with avoidable adverse drug events (ADEs). Historically, checklists such as Beers criteria (revised in 2003), which consisted of lists of drugs to be avoided in older people, were used. However, two recent large scale retrospective studies examined the association between PIMs identified through the Beers criteria and the incidence of ADEs, and found no statistically significant association.[1,3]

A research group at Cork University Hospital, Ireland, has recently devised and validated a new set of PIM criteria in older people called STOPP (Screening Tool of Older Persons' potentially inappropriate Prescriptions), published in 2011. This prospective study involved 600 consecutive patients aged 65 years or older. Their project highlighted that PIM in STOPP are significantly associated with avoidable ADEs in older people, and can cause or contribute to urgent hospitalization.[1,3]

In this project, STOPP criteria were used to screen the drug charts of HCE patients to identify potential PIMs, with an aim to reduce that number as low as possible $(<2 \%)$, through collective education 
of doctors, nurses, and pharmacists.

\section{Baseline measurement}

Through scrutinising medical notes and drug charts, we screened 148 medications on one HCE ward and identified eight (5.4\%) PIMs. When looking at the drug charts, we sought PIMs such as anticholinergics, long term opiates, and duplicate drug class prescriptions (see appendix 1), checked relevant blood results, and went through each patient's background and history to identify valid reasons for the patient to be on the PIM.

See supplementary file: ds6698.pdf - "Appendix 1: Summary of START and STOPP criteria"

\section{Design}

When considering the underlying cause of the problem, it became clear that all too often, drug charts would not be looked at in any depth during the busy ward round, and inappropriate medications would therefore not be identified. Rather than resorting to actively remembering to check the drug chart for every patient, which is often challenging under time and resource constraints, we designed a general ward round checklist to ensure the drug chart and other core daily information would be looked at during the ward round, including nutritional status and oral intake, fluid and stool charts, resuscitation status, and family discussion.

Inspired by the approach of our intensive care unit (ICU) colleagues, who rely on a comprehensive daily ward round checklist, we felt that a quick and concise checklist would help us to be systematic about rationalising drug charts and eradicating PIMs, as well as providing an equal and safe standard of care for our HCE patients.

\section{Strategy}

PDSA cycle 1 (1 to 5 December 2014)

The lesson we learnt from the baseline measurement was that HCE physicians were generally good at screening and stopping inappropriate medications, and there were very few PIM prescriptions on this HCE ward. With an aim to educate junior doctors and refresh the knowledge of senior members, these baseline measurements were presented at our weekly HCE teaching, defining PIMs, highlighting examples, and discussing how to justifiably withdraw these drugs in an appropriate way. We then carried out a further round of data collection; we screened 192 drugs and found 10 PIMs (5.2\%). Based on the lessons we had learnt, and after discussion with a HCE consultant, we developed a ward round checklist, and distributed the checklist among the junior doctors.

PDSA cycle 2 (15 to 20 December 2014)

The aim of this cycle was to reinforce the habit of screening the drug charts through the use of the HCE ward round (WR) checklist.
The checklist was designed and implemented on our consultant ward round twice a week for one week. The feedback from the junior doctors was that they found it difficult to follow the consultant ward round, keep up with the tasks collected during the ward round, and appropriately use the checklist in view of screening each drug chart for PIMs while ticking off the HCE WR checklist. The outcome after another cycle of data collection showed five PIMs out of 136 screened drugs (3.7\%).

\section{PDSA cycle 3 ( 1 to 5 January 2015)}

From the second PDSA cycle, we learnt an important lesson: enlisting help from other members of the multidisciplinary team would be beneficial. This time we included the ward pharmacist, who would check drug charts and patients' electrolytes on a daily basis if appropriate. We also distributed the PIMs list among the nursing staff, so that during their drug round, if they identified any PIMs, they could escalate this to a doctor for action to be taken accordingly. We identified seven PIMs out of 200 screened drugs (3.5\%).

PDSA cycle 4 (19 to 24 January 2015)

By maintaining constant awareness of PIMs through routine implementation of the WR checklist, the pharmacist's input, and the nurses' awareness during their drug round, cycle 4 showed a PIM of $3.7 \%$ (six PIMS out of 161 drugs).

PDSA cycle 5 (2 to 7 February 2015), 6 (23 to 28 February 2015), and 7 (9 to 14 March 2015)

A refresher presentation at our weekly departmental teaching showed a decrease in PIMs to $2.2 \%$ (three PIMS out of 133 ) in cycle 5. Cycle 6 showed a PIM rate of of $2.3 \%$ (three PIMs out of 126 ), and cycle 7 revealed a successful decrease to $1.5 \%$ (two PIMs out of 136).

\section{Results}

In our bi-weekly intervention measurement that took place for comparison over a four month period on HCE wards, we identified that outcomes improved progressively after each cycle, from a baseline of $5.4 \%$ of PIMs to $1.5 \%$ of PIMs during our last recorded cycle.

See graph 1 for details of the PIM reduction seen over the seven PDSA cycles.

See supplementary file: ds6699.docx - "Graph 1: showing PIM reduction over 7 PDSA cycles"

\section{Lessons and limitations}

We learnt a number of lessons from carrying out this project. Small steps of change are useful when testing an intervention on this scale, and fitting it around the demands of our clinical work as junior doctors. When designing an intervention, we needed to consider 
the wider range of healthcare professionals that the intervention could impact upon. Healthcare professionals operate in a high pressured workplace, which keeps them busy and makes them tired. It can be expected that an individual may occasionally make mistakes in this context; therefore, interventions should seek to eliminate the human factors which may have a bearing on the process.

By involving pharmacists in the quality project, we could ensure that even the drug charts sent to pharmacy during ward round times were checked on a daily basis. While on our ward there is a consultant ward round twice a week every week, some HCE patients, in particular on outlier wards, may not have the advantage of being seen by a senior doctor as frequently. Therefore, applying our checklist to these outlying patients may be more problematic. This could be overcome by enlisting the support of nurse managers or sisters for these outlier wards, to champion the use of this ward round checklist.

The core number of junior doctors joining the consultant ward round varies from day to day, given that on calls; nights; and annual, study, and sick leave will change the staffing level on the rota. While it may be fairly easy and safe to use a ward round checklist on a well staffed ward round, as one member can be in charge of the checklist and the drug chart review while others take care of other tasks on the round, this becomes very difficult if the ward is understaffed. It was apparent that for this system to be efficient, it requires a consistent and improved level of medical staffing. If this is not possible, we would also invite the ward pharmacist to be on the consultant ward round.

We put great effort into repeating our PDSA cycles with implemented changes, and proceeded with data collection on alternate weeks, working around our professional rota and other commitments in order to complete our QIP in our initial target time of four months. This project may have produced stronger results if the PDSA cycles had been continuous or weekly, but this was not possible due to clinical commitments and medical staffing constraints.

In this study, there were a small number of PIMs prior to implementing a ward round checklist on the HCE ward. Members of the clinical team were already aware of the need to improve prescribing practices, perhaps making them more receptive to our educational interventions. To extend this study, it would therefore be important to gather data from more wards, which would be more representative of the various practices of different HCE wards across the UK. This would also have the potential of providing us with larger numbers on which to perform analyses, to assess the statistical significance of reducing PIMs through ward round checklists.

\section{Conclusion}

Instituting these interventions, according to the PDSA cycles, had a positive impact among our multidisciplinary team on the ward, as it increased awareness of PIMs and their potential side effects among all the different team members. Together, we managed to improve safe prescribing and reduce the number of PIMs from the baseline measurements in a stepwise manner $(5.4 \%$ on baseline measurement, to $1.5 \%$ on PDSA cycle 7 ). In a busy environment such as a hospital ward, it is important to raise general awareness through educating all the team members, and making the intervention a shared responsibility between the prescriber, the pharmacist, and the drug administrator. A systematic approach to ward rounds with a ward round checklist offers a rigorous method to reduce the prevalence of PIMs, and the frequency of adverse drug events. This has important implications in the wider context of growing pressures to deliver higher standards of cost effective clinical care.

\section{References}

1. Hamilton H, Gallagher P, Ryan C, Byrne S, O'Mahony D. Potentially inappropriate medications defined by STOPP criteria and the risk of adverse drug events in older hospitalized patients. Arch Intern Med 2011;171(11):1013-9.

2. Trivalle $C$, Burlaud $A$, Ducimetière $P$, the IMEPAG Group. Risk factors for adverse drug events in hospitalized elderly patients: A geriatric score. Eur Geriatr Med 2011;2(5):284-9.

3. O'Mahony D, Gallagher P, Ryan C, et al. STOPP \& START criteria: A new approach to detecting potentially inappropriate prescribing in old age. Eur Geriatr Med 2010;1(1):45-51.

4. NHS Institute for Innovation and Improvement. Plan, Do, Study, Act (PDSA). 2008.

http://www.institute.nhs.uk/quality_and_service_improveme nt tools/quality and service improvement tools/plan do s tudy_act.html

5. Henley DB, Sundell KL, Sethuraman G, Schneider LS3. Adverse events and dropouts in Alzheimer's disease studies: What can we learn? Alzheimers Dement 2015;11(1):24-31.

\section{Declaration of interests}

Nothing to declare.

\section{Acknowledgements}

Adele Beck, Thomas Siese, Dr Paul Hancock.

\section{Ethical approval}

According to the policy activities that constitute research at Derriford Hospital, this work met criteria for operational improvement activities exempt from ethics review. We used the following criteria for determining if improvement activities require ethics review:

Policy criterion: The work is primarily intended to improve local care, not provide generalisable knowledge in a field of inquiry. 